\title{
Assessment of phytoplankton species in gut and feces of cultured tilapia fish in Egyptian fishponds: Implications for feeding and bloom control
}

\author{
Avaliação de espécies fitoplanctônicas no intestino e nas fezes de tilápia cultivada em \\ viveiros no Egito: implicações para a alimentação e controle da floração
}

\section{Zakaria Mohamed $^{1 *}$ (D), Zeinab Ahmed ${ }^{1}$ and Asmaa Bakr ${ }^{1}$}

${ }^{1}$ Department of Botany and Microbiology, Faculty of Science, Sohag University, Egypt

*e-mail: mzakaria_99@yahoo.com

Cite as: Mohamed, Z., Ahmed, Z. and Bakr, A. Assessment of phytoplankton species in gut and feces of cultured tilapia fish in Egyptian fishponds: Implications for feeding and bloom control. Acta Limnologica Brasiliensia, 2019, vol. 31, e27.

Abstract: Aim: This study was carried out to determine which phytoplankton species, as a natural food, can be ingested and digested by Nile tilapia (Oreochromis niloticus L.). Methods: During this study, phytoplankton in the gut contents of Nile tilapia collected from three fishponds in southern Egypt were investigated during the period Oct. 2012-Sep. 2013. Samples of tilapia fish were grown in aquarium containing filtered pond water to detect undigested phytoplankton species in the feces. Results: The majority of the phytoplankton found in the gut of Nile tilapia was Cyanobacteria (36-50\%) and Chlorophyta (27-38\%). Other groups such Diatoms, Euglenophyta and Dinophyta were also found but with lower percentages $(<19 \%)$. The most important and dominant phytoplankton species found in Tilapia gut were the potentially toxic cyanobacteria, Anabaena, Anabaenopsis, Cylindrospermopsis, Microcystis and Planktothrix. Only diatoms were recorded in the feces, indicating the ability of Tilapia to digest all phytoplankton except diatoms. Conclusions: The data of this study could be useful for biomanipulation of nuisance phytoplankton blooms in eutrophic aquacultures.

Keywords: cyanobacteria; diatoms; digestion; ingestion; tilapia.

Resumo: Objetivo: Nosso estudo visou determinar quais espécies de fitoplâncton, como alimento natural, podem ser ingeridas e digeridas pela tilápia do Nilo (Oreochromis niloticus L.). Métodos: Durante o estudo, coletamos e analisamos o fitoplâncton nos intestinos de tilápia do Nilo cultivadas em três viveiros de peixes no sul do Egito, durante o período de outubro de 2012 a setembro. 2013. Indivíduos de tilápias foram colocados em aquários contendo água filtrada para detectar espécies de fitoplâncton não digeridas nas fezes. Resultados: A maioria do fitoplâncton encontrado no intestino de tilápia do Nilo foi cianobactérias (36-50\%) e clorofíceas (27-38\%). Outros grupos, como Diatomáceas, Euglenophyta e Dinophyta, também foram encontrados em menores quantidades (<19\%). As espécies fitoplanctônicas mais importantes e dominantes encontradas no intestino de tilápia foram as cianobactérias potencialmente tóxicas, Anabaena, Anabaenopsis, Cylindrospermopsis, Microcystis e Planktothrix. Apenas as diatomáceas foram registradas nas fezes, indicando a capacidade da tilápia de digerir todo o fitoplâncton, exceto espécies deste grupo. Conclusáo: Os dados deste estudo podem ser úteis para a biomanipulação de proliferação de fitoplâncton em ambientes aquicolas eutróficos.

Palavras-chave: cianobactérias; diatomáceas; digestão; ingestâo; tilápia. 


\section{Introduction}

Grazing by higher trophic level organisms including fish and zooplankton can be a major contributor to the reduction of algal and cyanobacterial blooms (Mohamed \& Al-Shehri, 2013a). Nile tilapia (Oreochromis niloticus L.) is one of the most important tropical and subtropical freshwater fish that can feed on both phytoplankton and smaller zooplankton (Abdel-Tawwab, 2000). Phytoplankton cells could be used as a natural food of Tilapia fish (Fattah et al., 2008; Budihastuti et al., 2013), as they are rich in vitamin precursors, growth promoters and essential fatty acids (Awasthi et al., 2006). However, some phytoplankton groups, particularly cyanobacteria can produce toxins that may negatively affect fish health or accumulate in their tissues posing a risk to human health upon consumption of such contaminated fish (Mohamed et al., 2003; Mohamed, 2016). Therefore, the types of phytoplankton consumed by fish as a food should be determined before selecting them for fish feeds. Analysis of phytoplankton composition in fish gut contents is widely used to ascertain the food and feeding habit of fish (Nath et al., 2015). Furthermore, accurate identification of prey and feeding behavior provide the basis for understanding the trophic interactions in aquatic food webs (Zanden et al., 2000).

Several studies have shown that Tilapia has negative effects on cyanobacteria with high ingestion rates and digestion efficiencies ( $\mathrm{Lu}$ et al., 2006; Menezes et al., 2010; Salazar Torres et al., 2016). These studies therefore suggested that stocking tilapia is an effective way to control algal blooms in eutrophic waters. Conversely, some studies reported that Tilapia fish are not effective in reducing phytoplankton biomass through direct grazing, and can contribute with nutrient excretion to the increase of phytoplankton biomass in the aquatic systems (Silva et al., 2014). Furthermore, Semyalo et al. (2011) found no significant relationship between the contribution of phytoplankton in tilapia diet and microcystin concentrations in the water.

As phytoplankton is one of the main food items for Tilapia fish, we hypothesized that Nile Tilapia will decrease the phytoplankton biomass and composition in fishponds through direct herbivory (top-down control). We also hypothesized that Tilapia fish would not influence phytoplankton through nutrient recycling (bottom-up control), because our fishponds are eutrophic. The present study was carried out to determine the composition and abundance of phytoplankton species in fish pond waters as well as in the gut contents of Tilapia fish (Oreochromis niloticus) collected from these fishponds, to ascertain the preferences of fishes for phytoplankton species as food, and its capability of feeding on nuisance algae.

\section{Material and Methods}

\subsection{Description of study area}

This study was conducted in three fishponds located in Sohag governorate during the period Oct. 2012 - Sep2013 (fishponds 1, 2 and 3). Fishpond 1 is located at $26^{\circ} 27 \mathrm{~N}$, and $31^{\circ} 40 \mathrm{E}$. It receives water from Nile River and agricultural drains with about $3 \mathrm{~m}$ depth. Fishpond 2 is located at $26^{\circ} 27 \mathrm{~N}$, and $\left.31^{\circ} 49 \mathrm{E}\right)$. This fishpond receives water from the Nile River with about $4 \mathrm{~m}$ depth. Fishpond 3 is located at $26^{\circ} 36 \mathrm{~N}$, and $31^{\circ} 4383 \mathrm{E}$ ). It receives water from the Nile River, with about $3.5 \mathrm{~m}$ depth. In a parallel study by us, these fishponds were regarded as hypertotrophic (chl. a concentrations exceeded $75 \mu \mathrm{g} \mathrm{L}^{-1}$ ) with physico-chemical parameters characterized by high temperature, moderate $\mathrm{pH}$ and high nutrient concentrations, particularly during warm months (Mohamed \& Bakr, 2018). The fish densities in these fishponds ranged from of 7 to $9 \mathrm{fish} / \mathrm{m}^{3}$.

\subsection{Phytoplankton analysis in gut contents and feces.}

Fish pond water and fish samples tilapia (Oreochromis niloticus) were collected monthly from three fish farms during the period from October 2012 to September2013 for 12 months except fishpond 1 for only nine months because no water samples collected at that time as the pond was dried up due to the Nile's low water level. The live fish samples were washed with distilled water to remove phytoplankton attached to their surfaces. Fishes were weighed, scarified and dissected. The gut of each fish was removed and fixed with $1 \mathrm{~mL}$ of Lugol's solution for microscopic examination. In order to test the digestibility of phytoplankton species by tilapia fish, about 10 tilapias were collected from fishpond 3 on a certain sampling occasion, and introduced into aquarium containing strained fishpond water by filtering through a $20 \mu \mathrm{m}$ plankton net following the method of Ping \& Jiankang (1994).The feces were collected immediately after digestion by means of a pipette, and washed twice carefully in distilled water. They were homogenized with a stirrer for a few minutes, and fixed in Lugol's iodine. Phytoplankton species in fishpond waters, fish gut and feces were identified 
based on morphological characteristics according to Prescott (1978). The counts of these species were performed using Sedgwick-Rafter under a binocular inverted microscope.

\section{Results}

The species composition of phytoplankton recorded in fishpond waters and the gut of Tilapia fish caught from these fishponds during the period Oct. 2012-Sep.2013 is shown in tables 1-4. Fifty four species of different groups were identified in Tilapia fish gut during the study period. Of which, 20 species belonging to Cyanobacteria, 18 to Chlorophyta,
7 to Bacillariophyta, 4 to Dinoflagellates, 2 to Charophyta and 2 to Euglenophyta. The biomass composition of phytoplankton (based on cell count) in the fish gut varied significantly among fishponds and study months. Cyanobacteria and Chlorophyta constituted the highest percentages of phytoplankton biomass along the study period $(36-50 \% \& 27-38 \%$, respectively). Other phytoplankton groups such as Bacillariophyta, Dinophyta, Charophyta and Euglenophyta were found in the fish gut with lower percentages, where they constituted $12-18.9 \%$, $8 \%, 5-6 \%, 2-5 \%$ of total phytoplankton biomass, respectively (Tables 1-3). The phytoplankton species in the fish gut varied significantly among

Table 1. Phytoplankton species composition (cells $\times \mathbf{x 1 0}^{6} \mathbf{L}^{-1}$ ) in fishponds used in the present study.

\begin{tabular}{|c|c|c|c|}
\hline $\begin{array}{r}\text { Algal species } \\
\end{array}$ & Fishpond 1 & Fishpond 2 & Fishpond 3 \\
\hline \multicolumn{4}{|l|}{ Cyanobacteria } \\
\hline Anabaena affinis Lemmermann & $1-100$ & - & - \\
\hline Anabaenopsis circularis (G.S.West) & - & $0.1-1$ & - \\
\hline Aphanizomenon gracile Lemmermann & - & $0.1-0.8$ & $0.3-1$ \\
\hline Aphanocapsa rivularis (Carmichael) & - & $0.1-0.5$ & $1-2$ \\
\hline Chroococcus minimus (Keissler) & $2-3$ & $0.2-1.1$ & $0.5-1$ \\
\hline Coelosphaerium kuetzingianum Nägeli & $1-5$ & $0.1-1$ & 2 \\
\hline Cylindrospermopsis catemaco Komár & - & $0.1-1$ & $12-24$ \\
\hline C. philippinensis Taylor & - & $0.2-1$ & $11-20$ \\
\hline Cylindrospermopsis raciborskii (Woloszynska) & - & $0.3-1$ & $12-24$ \\
\hline Gomphosphaeria aponina Kützing & - & $0.01-0.1$ & 14-32 \\
\hline Lyngbya wollei (Farlow ex Gomont) & $1-80$ & - & - \\
\hline Merismopedia minima G.Beck & 20 & $0.1-0.5$ & - \\
\hline Merismopedia tenuissima Lemmermann & $0.5-20$ & $0.2-0.5$ & $4-24$ \\
\hline Microcystis aeruginosa (Kützing) & $0.5-1$ & $0.3-0.5$ & $3-54$ \\
\hline Oscillatoria formosa Bory ex Gomon & 1 & - & - \\
\hline Oscillatoria limnetica Lemmermann & 1 & $0.1-0.4$ & $2-12$ \\
\hline Planktolyngbya limnetica (Lemmermann) & 2 & - & - \\
\hline Pseudanabaena catenata Lauterborn & - & $0.2-0.7$ & - \\
\hline Planktothrix agardhii (Gomont) & $1-110$ & - & - \\
\hline Spirulina abbreviata Lemmermann & $0.5-9$ & 0.5 & $1-10$ \\
\hline Synechocystis aquatilis Sauvageau & - & $1-3$ & $1-11$ \\
\hline \multicolumn{4}{|l|}{ Chlorophyta } \\
\hline Actinastrum hantzschii Lagerheim & $2-56$ & $11-34$ & $8-77$ \\
\hline Ankistrodesmus gracilis (Reinsch) & $10-76$ & $34-54$ & $4-76$ \\
\hline Chlamydomonas reinhardtii P.A.Dangeard & $22-55$ & 23 & $6-76$ \\
\hline Chlorella vulgaris Beyerinck & $22-88$ & $6-7$ & 88 \\
\hline Chlorococcum lobatum (Korshikov) & $11-54$ & $6-90$ & $8-67$ \\
\hline Cladophora aegagropila (Linnaeus) & $36-98$ & $6-9$ & $3-5$ \\
\hline Cosmarium abbreviatum Raciborski & $13-76$ & 8-81 & $4-65$ \\
\hline Crucigenia fenestrata (Schmidle) & $12-32$ & $4-6$ & $56-76$ \\
\hline Eudorina elegans Ehrenberg & $32-34$ & $8-89$ & 32 \\
\hline Kirchneriella lunaris (Kirchner) & 54 & $6-9$ & 58 \\
\hline Monoraphidium arcuatum (Korshikov) & $10-45$ & - & 4 \\
\hline Ochromonas tuberculata D.J.Hibberd & - & $34-65$ & $4-54$ \\
\hline Pandorina morum (O.F.Müller) & 5 & $7-70$ & $5-63$ \\
\hline Pediastrum duplex Meyen & $6-12$ & 8 & $1-8$ \\
\hline Scenedesmus ellipsoideus Chodat & $4-98$ & $3-98$ & $2-11$ \\
\hline Staurastrum anatinum Cooke \& Wills & $3-65$ & $5-78$ & $3-54$ \\
\hline Tetraëdron minimum (A.Braun) & $4-11$ & $1-98$ & - \\
\hline
\end{tabular}


Table 1. Continued...

\begin{tabular}{lccc}
\hline \multicolumn{1}{c}{ Algal species } & Fishpond 1 & Fishpond 2 & Fishpond 3 \\
\hline Euglenophyta & & & $4-65$ \\
Euglena agilis H.J.Carter & $6-23$ & - & $10-54$ \\
Phacus acuminata Kiss & - & & $2-8$ \\
Bacillariophyta & & $3-7$ & 34 \\
Cyclotella sp. & 7 & - & $5-65$ \\
Cymbella sp. & - & $5-7$ & $4-11$ \\
Fragillaria sp. & $5-65$ & $2-8$ & $1-87$ \\
Melosira sp. & $4-14$ & - & $4-89$ \\
Navicula sp. & $6-87$ & $3-9$ & $3-98$ \\
Nitzschia $s p$. & $2-45$ & - & 3 \\
Tribonema $s p$. & $4-76$ & - & - \\
Tabillaria $s p$. & $4-45$ & - & 12 \\
Charophyta & & - & 23 \\
Cloesteruim $s p$. & $7-56$ & - & - \\
Spirogyra sp. & 45 & - & 67 \\
Dinoflagellates & & - & $9-11$ \\
Ceratinum $s p$. & 22 & - & \\
Gymnodinium $s p$. & 9 & - & \\
Katodinium $s p$. & - & & \\
Peridinium $s p$. & 12 & - & \\
\hline
\end{tabular}

Table 2. Analysis of different phytoplankton groups identified in fish gut (cells $\times 10^{6}$ gut $^{-1}$ ) from fishpond 1 during the study period Oct. 2012- Sept. 2013.

\begin{tabular}{|c|c|c|c|c|c|c|c|c|c|c|c|c|}
\hline Algal species & Oct. & Nov. & Dec. & Jan. & Feb. & Mar & April & May & June & July & Aug & Sep. \\
\hline \multicolumn{13}{|l|}{ Cyanobacteria } \\
\hline Anabaena affinis Lemm & 1.3 & 1 & - & - & - & - & 0.5 & 0.8 & 0.86 & 1.5 & 3 & 4.2 \\
\hline Chroococcus minimus Keissl & 3.2 & - & - & - & - & - & - & - & - & - & - & - \\
\hline Coelosphaerium kuetzingianum Näg & 4 & 3 & - & - & - & - & - & - & - & - & - & - \\
\hline Cylindrospermopsis raciborskii Wol & 2.2 & - & - & - & - & - & - & - & - & - & - & - \\
\hline Gomphosphaeria aponina Kützing & 1.8 & 2 & - & - & - & - & - & - & - & - & - & - \\
\hline Lyngbya wollei Farlow ex Gomont & - & - & & - & - & - & 0.07 & 0.78 & 0.9 & 1 & 1.3 & 3 \\
\hline Merismopedia minima G.Beck & 3.1 & 3 & - & - & - & - & - & - & - & - & - & - \\
\hline Merismopedia tenuissima Lemm & - & 4 & 1 & - & - & - & - & - & - & - & - & 23 \\
\hline Microcystis aeruginosa (Kützing) & 3 & - & - & & - & - & - & - & - & - & - & 2 \\
\hline Oscillatoria formosa Bory ex Gomon & 4 & - & - & - & - & - & - & - & - & - & - & - \\
\hline Oscillatoria limnetica Lemm & 5 & 2 & - & - & - & & - & - & - & - & - & 12 \\
\hline Planktolyngbya limnetica Lemm & - & - & 6 & - & - & - & - & - & - & - & - & - \\
\hline Planktothrix agardhii (Gomont) & 4 & - & - & - & - & - & 0.4 & 0.3 & 0.7 & 3 & 5 & 7 \\
\hline Spirulina abbreviata Lemm & - & - & - & - & - & - & - & - & 1 & 4 & 6 & 8 \\
\hline Synechocystis aquatilis Sauvageau & 12 & - & - & - & - & - & - & - & - & - & - & - \\
\hline \multicolumn{13}{|l|}{ Chlorophyta } \\
\hline Actinastrum hantzschii Lagerheim & 43 & - & - & - & 23 & 43 & 45 & 33 & - & - & - & - \\
\hline Ankistrodesmus gracilis (Reinsch) & - & - & 43 & 12 & 45 & 56 & 42 & 22 & - & - & - & - \\
\hline $\begin{array}{l}\text { Chlamydomonas reinhardtii } \\
\text { Dangeard }\end{array}$ & - & - & - & - & - & - & 45 & 31 & - & - & - & - \\
\hline Chlorella vulgaris Beyerinck & - & 3 & - & 67 & 30 & 18 & 19 & 20 & - & - & 21 & - \\
\hline Chlorococcum lobatum (Korshikov) & - & - & - & 34 & 55 & 34 & 22 & 18 & - & - & - & - \\
\hline Cladophora aegagropila (Linnaeus) & - & - & - & - & 7 & 4 & 2 & 5 & - & - & - & - \\
\hline Cosmarium abbreviatum Raciborski & 5 & - & 3 & 4 & 21 & 4 & 2 & - & - & - & - & - \\
\hline Crucigenia fenestrata (Schmidle) & 3 & 2 & 2 & - & - & - & - & - & - & - & - & - \\
\hline Kirchneriella lunaris (Kirchner) & - & - & 2 & - & - & - & - & - & - & - & - & - \\
\hline Monoraphidium arcuatum Korshikov & - & 11 & - & - & - & - & - & - & - & - & - & - \\
\hline Pandorina morum (O.F.Müller) & - & 34 & 53 & - & - & - & - & - & - & - & - & - \\
\hline Pediastrum duplex Meyen & 2 & 7 & 44 & 59 & 52 & 65 & - & - & - & - & 2 & - \\
\hline Scenedesmus ellipsoideus Chodat & 3 & 5 & 11 & - & - & - & 2 & - & - & 1 & 0.9 & - \\
\hline Staurastrum anatinum Cooke \& Wills & - & 3 & 6 & - & - & - & - & 2 & - & - & - & 11 \\
\hline Tetraëdron minimum (A.Braun) & 2 & 1 & - & 21 & 11 & 17 & 10 & - & - & - & - & 15 \\
\hline
\end{tabular}


Table 2. Continued...

\begin{tabular}{|c|c|c|c|c|c|c|c|c|c|c|c|c|}
\hline Algal species & Oct. & Nov. & Dec. & Jan. & Feb. & Mar & April & May & June & July & Aug & Sep. \\
\hline \multicolumn{13}{|l|}{ Euglenophyta } \\
\hline Euglena agilis H.J.Carter & 23 & 11 & 3 & - & - & - & 11 & - & 12 & - & 2 & - \\
\hline \multicolumn{13}{|l|}{ Bacillariophyta } \\
\hline Cyclotella sp. & 22 & 7 & 19 & - & - & - & 21 & - & - & - & 4 & - \\
\hline Fragillaria $s p$. & - & - & - & - & - & - & - & - & 12 & - & - & - \\
\hline Melosira sp. & - & - & 34 & - & - & - & 23 & 5 & 11 & - & 24 & 35 \\
\hline Navicula sp. & 11 & 31 & 33 & - & - & - & - & 50 & 45 & 56 & 67 & 51 \\
\hline Nitzschia sp. & 21 & 43 & 5 & - & - & - & 39 & 28 & 41 & 53 & 67 & - \\
\hline Tribonema sp. & 23 & 11 & 32 & - & - & - & - & - & 10 & - & 12 & - \\
\hline Tabillaria sp. & - & 23 & 43 & - & - & - & - & - & 12 & - & 9 & - \\
\hline \multicolumn{13}{|l|}{ Charophyta } \\
\hline Cloesteruim sp. & - & - & - & - & - & - & 34 & - & - & 11 & - & - \\
\hline Spirogyra sp. & - & - & - & - & - & - & - & 67 & 34 & 4 & 11 & 2 \\
\hline \multicolumn{13}{|l|}{ Dinoflagellates } \\
\hline Ceratinum sp. & - & - & - & - & - & - & 21 & - & - & - & - & - \\
\hline Gymnodinium sp. & - & - & 1 & - & - & - & - & - & - & - & - & - \\
\hline Peridinium sp. & 21 & 11 & 23 & - & - & - & 41 & - & - & - & 9 & - \\
\hline
\end{tabular}

Table 3. Analysis of different phytoplankton groups identified in fish gut (cells $\times 10^{6}$ gut $\left.^{-1}\right)$ from fishpond 2 during the study period Oct. 2012- Sept. 2013.

\begin{tabular}{|c|c|c|c|c|c|c|c|c|c|c|c|c|}
\hline Algal species & Oct & Nov & Dec & Jan & Feb & Mar. & Apr & May & Jun & Jul & Aug & Sep \\
\hline \multicolumn{13}{|l|}{ Cyanobacteria } \\
\hline Anabaenopsis circularis (G.S. West) & 33 & - & - & - & - & - & - & - & - & 19 & 32 & 52 \\
\hline Aphanocapsa rivularis Carmichael & 22 & - & - & - & - & - & 12 & 43 & 65 & 45 & 77 & 78 \\
\hline Chroococcus minimus Keissl & 54 & - & - & - & - & - & - & - & - & - & - & - \\
\hline Coelosphaerium kuetzingianum Näg & 76 & - & - & - & - & - & - & - & - & 59 & - & 40 \\
\hline Cylindrospermopsis catemaco Komárk & 60 & - & - & - & - & - & - & - & - & - & - & 87 \\
\hline C. philippinensis W.R.Taylor & - & - & - & - & - & - & - & - & - & 54 & 45 & 77 \\
\hline C. raciborskii Wol. & 23 & - & - & - & - & - & - & - & 43 & 67 & 98 & 56 \\
\hline Gomphosphaeria aponina Kütz & 66 & - & - & - & - & - & - & - & - & - & - & - \\
\hline Merismopedia minima G.Beck & 67 & 100 & 57 & 65 & 45 & 33 & 69 & - & - & - & - & 87 \\
\hline Merismopedia tenuissima Lemm & 39 & - & - & - & - & - & - & - & - & - & - & - \\
\hline Microcystis aeruginosa Kütz & 43 & 56 & 34 & 12 & - & - & - & - & - & - & - & - \\
\hline Oscillatoria limnetica Lemm & - & - & - & - & - & - & - & 67 & - & - & - & 45 \\
\hline Pseudanabaena catenata Lauterborn & - & - & - & 54 & 33 & 23 & - & - & - & - & - & 55 \\
\hline Spirulina abbreviata Lemm & - & - & - & - & - & - & - & - & - & - & 65 & - \\
\hline Synechocystis aquatilis Sauvageau & 8 & 54 & 45 & 80 & 19 & & - & - & - & - & - & - \\
\hline \multicolumn{13}{|l|}{ Chlorophyta } \\
\hline Actinastrum hantzschii Lagerheim & - & - & 43 & - & 56 & - & - & - & - & - & - & - \\
\hline Ankistrodesmus gracilis Reinsch & - & 12 & 43 & - & 66 & 78 & 45 & 55 & - & - & - & - \\
\hline Chlamydomonas reinhardtii Dang & & & 34 & - & - & - & - & - & - & - & - & - \\
\hline Chlorella vulgaris Beyerinck & - & 43 & - & 33 & 32 & - & 33 & 23 & - & - & - & - \\
\hline Chlorococcum lobatum (Korshikov) & - & - & - & - & 43 & - & 12 & - & - & - & - & - \\
\hline Cladophora aegagropila (Linnaeus) & - & - & - & - & - & - & 12 & - & - & - & - & - \\
\hline Cosmarium abbreviatum Raciborski & - & 43 & - & - & - & - & 32 & 43 & - & - & - & - \\
\hline Crucigenia fenestrata (Schmidle) & 12 & 22 & 32 & - & - & 19 & - & - & - & - & - & - \\
\hline Eudorina elegans Ehrenberg & - & - & - & - & 34 & - & - & - & - & - & - & - \\
\hline Kirchneriella lunaris (Kirchner) & - & - & - & - & - & 32 & - & - & - & - & - & - \\
\hline Monoraphidium arcuatum (Korshikov) & - & - & - & - & - & 7 & - & - & - & - & - & - \\
\hline Ochromonas tuberculata D.J.Hibberd & - & - & - & - & - & 12 & - & - & - & - & - & - \\
\hline Pandorina morum (O.F.Müller) & - & - & - & - & 32 & - & 4 & 5 & - & - & - & - \\
\hline Pediastrum duplex Meyen & - & 31 & 43 & - & - & 55 & 3 & 5 & 1 & - & - & - \\
\hline Scenedesmus ellipsoideus Chodat & 3 & 20 & 4 & 43 & 5 & 0.8 & - & & - & 0.6 & - & - \\
\hline Staurastrum anatinum Cooke \& Wills & 12 & 32 & 11 & - & 34 & 43 & 33 & 54 & - & - & - & 20 \\
\hline Tetraëdron minimum (A.Braun) & 22 & 32 & 23 & - & 43 & 4 & - & - & - & - & - & 5 \\
\hline
\end{tabular}


Table 3. Continued...

\begin{tabular}{lcccccccccccc}
\hline \multicolumn{1}{c}{ Algal species } & Oct & Nov & Dec & Jan & Feb & Mar. & Apr & May & Jun & Jul & Aug & Sep \\
\hline Bacillariophyta & & & & & & & & & & & & \\
Cymbella $s p$. & - & - & - & - & - & - & - & 12 & - & - & - & - \\
Fragillaria $s p$. & - & - & - & & 100 & 40 & - & - & - & - & - & - \\
Melosira $s p$. & 28 & 56 & 57 & 40 & 30 & 56 & 23 & 34 & 4 & - & 2 & - \\
Nitzschia $s p$. & - & 23 & 50 & 49 & - & 38 & - & 56 & 87 & 67 & 72 & - \\
\hline
\end{tabular}

Table 4. Analysis of different phytoplankton groups identified in fish gut (cells $\times 10^{6}$ gut $\left.^{-1}\right)$ from fishpond 3 during the study period Oct. 2012- Sept. 2013.

\begin{tabular}{|c|c|c|c|c|c|c|c|c|c|c|c|c|}
\hline Algal species & Oct & Nov & Dec & Jan & Feb & Mar & Apr & May & Jun & Jul & Aug & Sep \\
\hline \multicolumn{13}{|l|}{ Cyanobacteria } \\
\hline Aphanocapsa rivularis Carmichael & - & - & 34 & 34 & - & - & - & - & - & - & - & - \\
\hline Chroococcus minimus Keissl. & 23 & - & - & - & - & - & - & - & - & - & - & 56 \\
\hline $\begin{array}{l}\text { Coelosphaerium kuetzingianum } \\
\text { Näg. }\end{array}$ & 34 & - & - & - & - & - & - & - & - & - & - & - \\
\hline $\begin{array}{l}\text { Cylindrospermopsis catemaco } \\
\text { Komár }\end{array}$ & 25 & - & - & - & - & - & - & - & 45 & 55 & 63 & 40 \\
\hline C. philippinensis Taylor & 34 & - & - & - & - & - & - & - & - & 23 & 43 & 30 \\
\hline C. raciborskii Wol. & 23 & - & - & - & - & - & - & - & 24 & 43 & 55 & 20 \\
\hline Gomphosphaeria aponina Kützing & 100 & - & - & 2 & - & - & - & - & - & - & - & 63 \\
\hline Merismopedia tenuissima Lemm. & 200 & - & 27 & - & - & - & - & - & - & - & - & 88 \\
\hline Microcystis aeruginosa (Kützing) & 34 & 42 & 23 & 7 & 1 & - & 2 & 2 & 17 & 54 & 65 & 40 \\
\hline Oscillatoria limnetica Lemm & 45 & - & - & - & - & - & - & - & - & 13 & 17 & 20 \\
\hline Spirulina abbreviata Lemm & - & - & - & - & - & & - & 13 & - & - & - & 21 \\
\hline $\begin{array}{l}\text { Synechocystis aquatilis } \\
\text { Sauvageau }\end{array}$ & 11 & - & - & - & - & - & - & - & - & - & - & 23 \\
\hline \multicolumn{13}{|l|}{ Chlorophyta } \\
\hline Actinastrum hantzschii Lagerheim & 22 & - & - & - & 34 & 55 & - & - & - & - & - & 3 \\
\hline Ankistrodesmus gracilis (Reinsch) & 18 & 20 & 30 & 48 & 100 & 87 & 75 & 65 & - & - & - & 40 \\
\hline Chlorella vulgaris Beyerinck & - & - & - & - & - & - & - & - & - & - & - & - \\
\hline $\begin{array}{l}\text { Chlorococcum lobatum } \\
\text { (Korshikov) }\end{array}$ & - & - & - & - & 34 & - & - & - & - & - & - & - \\
\hline $\begin{array}{l}\text { Cladophora aegagropila } \\
\text { (Linnaeus) }\end{array}$ & - & - & - & - & - & 54 & - & - & - & - & - & - \\
\hline $\begin{array}{l}\text { Cosmarium abbreviatum } \\
\text { Raciborski }\end{array}$ & - & - & - & - & - & - & - & - & - & - & - & 24 \\
\hline Crucigenia fenestrata (Schmidle) & - & - & - & 16 & 51 & 30 & - & - & - & - & - & - \\
\hline Eudorina elegans Ehrenberg & - & - & - & - & - & 33 & - & - & - & - & - & - \\
\hline Kirchneriella lunaris (Kirchner) & - & 21 & - & - & 32 & 54 & - & - & - & - & - & - \\
\hline $\begin{array}{l}\text { Monoraphidium arcuatum } \\
\text { Korshikov }\end{array}$ & 12 & 32 & 55 & & 52 & 60 & 67 & 32 & - & - & - & 17 \\
\hline $\begin{array}{l}\text { Ochromonas tuberculata } \\
\text { D.J.Hibberd }\end{array}$ & 14 & 3 & 42 & 5 & 30 & 67 & - & - & - & - & - & - \\
\hline Pandorina morum (O.F.Müller) & 5 & 21 & 3 & 55 & 87 & 53 & 77 & 3 & 0.7 & 0.9 & 0.3 & 1 \\
\hline Pediastrum duplex Meyen & & - & - & - & 54 & - & - & 78 & - & - & - & 87 \\
\hline Scenedesmus ellipsoideus Chodat & 54 & - & 67 & - & - & 56 & 75 & 45 & 32 & - & - & 55 \\
\hline $\begin{array}{l}\text { Staurastrum anatinum Cooke \& } \\
\text { Wills }\end{array}$ & 32 & 19 & - & - & - & 56 & - & - & - & - & - & - \\
\hline \multicolumn{13}{|l|}{ Euglenophyta } \\
\hline Euglena agilis Carter & 12 & 34 & 9 & 4 & 11 & 34 & 2 & - & 4 & 1 & 4 & 3 \\
\hline Phacus acuminata Kiss & 11 & 8 & - & - & - & - & 7 & - & - & - & - & - \\
\hline \multicolumn{13}{|l|}{ Bacillariophyta } \\
\hline Cyclotella sp. & - & 54 & 45 & 35 & 65 & 54 & 22 & 11 & - & - & - & - \\
\hline Cymbella sp. & - & - & - & - & - & 23 & - & - & - & - & - & - \\
\hline Fragillaria sp. & - & - & - & - & 45 & 56 & - & - & - & - & - & - \\
\hline Melosira sp. & - & 11 & 12 & 23 & 13 & 34 & 34 & 20 & 34 & 67 & - & - \\
\hline Navicula sp. & 8 & 34 & 54 & 2 & 15 & 65 & 24 & 23 & 42 & - & 32 & 32 \\
\hline Nitzschia sp. & 11 & 32 & 24 & 53 & 11 & 19 & 54 & 23 & 43 & - & 2 & 1 \\
\hline Tribonema sp. & 3 & 4 & 0.9 & - & 2 & 2 & - & 1 & - & 4 & 13 & 4 \\
\hline \multicolumn{13}{|l|}{ Dinoflagellates } \\
\hline Gymnodinium sp. & 3 & - & - & - & - & - & - & - & - & - & - & - \\
\hline Katodinium sp. & 2 & - & - & - & & - & - & - & - & - & - & - \\
\hline Peridinium sp. & 23 & 34 & 12 & 32 & 22 & 24 & 24 & - & 32 & - & - & 21 \\
\hline
\end{tabular}


study months, and dominated by the chlorophytes (e.g. Actinastrum, Ankistrodesmus, Chlamydomonas, Chlorella, Chlorococcum, Pediastrum and Scenedesmus) in winter, the cyanobacteria (Anabaena, Anabaenopsis, Cylindrospermopsis, Microcystis and Planktothrix) in summer and autumn, the Euglenophyta (Euglena) in autumn and winter, the diatoms (Cyclotella, Melosira, Navicula and Nitzschia) in spring and the dinoflagellate (Peridinium) in autumn. Results of microscopic investigation of phytoplankton composition in fish feces along one week compared to those in gut contents, revealed that all phytoplankton cells of cyanobacteria, chlorophyta, dinophyta, disappeared from the gut and not found in the feces (Table 4,5). In contrast, all diatom species disappeared from the gut and detected in the feces (Table 5).

Table 5. Phytoplankton species in gut contents of tilapia fish collected from fishpond 3, and in feces after growing in aquarium containing filtered pond water for one week.

\begin{tabular}{|c|c|c|}
\hline Phytoplankton species & $\begin{array}{c}\text { Gut } \\
\text { contents }\end{array}$ & Feces \\
\hline \multicolumn{3}{|l|}{ Cyanobacteria } \\
\hline $\begin{array}{l}\text { Chroococcus minimus } \\
\text { Keissler }\end{array}$ & + & - \\
\hline $\begin{array}{l}\text { Cylindrospermopsis } \\
\text { raciborskii (Woloszynska }\end{array}$ & + & - \\
\hline $\begin{array}{l}\text { Gomphosphaeria aponina } \\
\text { Kützing }\end{array}$ & + & - \\
\hline $\begin{array}{l}\text { Merismopedia tenuissima } \\
\text { Lemmermann }\end{array}$ & + & - \\
\hline $\begin{array}{l}\text { Microcystis aeruginosa } \\
\text { (Kützing) }\end{array}$ & + & - \\
\hline $\begin{array}{l}\text { Oscillatoria limnetica } \\
\text { Lemmermann }\end{array}$ & + & - \\
\hline $\begin{array}{l}\text { Pseudanabaena catenata } \\
\text { Lauterborn }\end{array}$ & + & - \\
\hline $\begin{array}{l}\text { Spirulina abbreviata } \\
\text { Lemmermann }\end{array}$ & + & - \\
\hline $\begin{array}{l}\text { Synechocystis aquatilis } \\
\text { Sauvageau }\end{array}$ & + & - \\
\hline \multicolumn{3}{|l|}{ Chlorophyta } \\
\hline $\begin{array}{l}\text { Actinastrum hantzschii } \\
\text { Lagerheim }\end{array}$ & + & - \\
\hline $\begin{array}{l}\text { Ankistrodesmus gracilis } \\
\text { (Reinsch) }\end{array}$ & + & - \\
\hline Pediastrum duplex Meyen & + & - \\
\hline $\begin{array}{l}\text { Scenedesmus ellipsoideus } \\
\text { Chodat }\end{array}$ & + & - \\
\hline \multicolumn{3}{|l|}{ Euglenophyta } \\
\hline Euglena agilis Carter & + & - \\
\hline \multicolumn{3}{|l|}{ Bacillariophyta } \\
\hline Navicula sp. & + & + \\
\hline Nitzschia sp. & + & + \\
\hline \multicolumn{3}{|l|}{ Dinophyta } \\
\hline Peridinium sp. & + & - \\
\hline
\end{tabular}

\section{Discussion}

Consistent with our hypothesis, Tilapia fed and reduced phytoplankton biomass in fishponds. The results showed that most phytoplankton species present in fishpond waters are pre-dominant in the gut contents and preferred food for Tilapia fish. Cyanobacteria and chlorophytes constituted the most phytoplankton groups in Tilapia fish gut during the study period. These results are thus in agreement with those obtained by Turker et al. (2003) reporting that Nile tilapia is more effective for filtering green algae and cyanobacteria from water sources. Salazar Torres et al. (2016) also analyzed tilapia gut contents and confirmed that cyanobacteria were a major component of its diet. More recently, Osti et al. (2018) found that cyanobacterial biomass in fishponds of a Nile tilapia production system in Brazil, was 2 to 3 times more than that in the system without tilapia, indicating the feeding of Tilapia on cyanobacteria. Additionally, euglenophytes and dinophytes and a little bit diatoms, were also detected in Tilapia fish gut in our study. Quite similar results were previously obtained by Abdel-Tawwab \& Sweilum (2003) for Nile tilapia (Oreochromis niloticus) cultured in earthen ponds. Abdel-Tawwab \& El-Marakby (2004) also showed that Cyanobacteria and Euglenophyceae were the most food found in the stomach of Tilapia. In the present study, the most common species found in Tilapia fish gut including the green algae (Ankistrodesmus, Scenedesmus Actinastrum), the cyanobacteria (Anabaena, Microcystis, Oscillatoria), the diatoms (Navicula, Nitzschia), and the euglenophytes (Euglena, Phacus) were also recorded previously in Nile tilapia stomachs from the Nile River (Abdelghany, 1993) and fishponds in Egypt (Abdel-Tawwab, 2000; Abdel-Tawwab \& Sweilum, 2003).

Potentially toxic cyanobacteria such as Microcystis and Anabaena were recognized as the most dominant phytoplankton ingested by the Nile tilapia in different lakes (Bwanika et al., 2006; Semyalo et al., 2011). In addition to these species, our study recorded other cyanobacterial species in Tilapia fish gut including Anabaenopsis, Cylindrospermopsis and Merismopedia. These species were previously reported as toxin producers (Mohamed \& Al-Shehri, 2009, 2010, 2013b; Mohamed \& Bakr, 2018). The abundance of these species in fish gut seems dependant on the density of these species in the environment and the condition of fish (Xie et al., 2001; Abdel-Tawwab \& Sweilum, 2003). This could be true for our results, as these 
species were abundant in Tilapia fish gut and in fishpond waters as well. This means that Tilapia can feed on all cyanobacteria without any avoidance for toxic species. In this respect, Lu et al. (2006) found that stocking Tilapia fish played an important role in reducing the biomass of bloom-forming species such as Oscillatoria princes and $M$. aeruginosa and Merismopedia tenuissima in Lake Yuehu, Ningbo. Salazar Torres et al. (2016) also provided evidence that Tilapia has the potential to reduce approximately $60 \%$ of cyanobacteria community in eutrophic reservoirs.

Interestingly, our study showed that Tilapia could ingest different morphological forms of phytoplankton including filamentous, colonial and single celled forms. This supports the fact that tilapia is a generalist filter feeder, which is capable of efficiently ingesting small and large phytoplankton (Turker et al., 2003). More recently, Rivera Vasconcelos et al. (2018) also reported that the Nile tilapia can suppress phytoplankton biomass in tropical lakes and reservoirs, with higher efficiency at feeding on larger algal and cyanobacterial forms than on small ones. Ingestion of phytoplankton by fish does not imply digestion and assimilation, which usually aided by two mechanisms including physical grinding of phytoplankton cells between two pharyngeal plates of fine teeth, and a stomach $\mathrm{pH}$ below 1.5 which lyses algal cell walls (TeichertCoddingeton et al., 1997; Xie et al., 2001). Here in the present study, we examined the feces of Tilapia fish to determine its ability to digest phytoplankton species ingested. The results showed that except diatoms, all phytoplankton cells detected in Tilapia fish gut were not found in the feces. This indicates that Tilapia fish were able to digest cyanobacteria, green algae, dinoflagellates and euglenophytes, but not able to digest diatom cells. This agrees with the results of many studies reporting that Adult Tilapia fish have a $\mathrm{pH}$ of about 1.4 in their digestive organs, so most phytoplankton cells can be digested (Tavera, 1996; Komarkova \& Tavera, 2003). Specifically, Lu et al. (2006) showed that Tilapia is among the very few fish species which are capable of digesting cyanobacteria.

Other studies showed the ability of Tilapia to assimilate bioactive compounds produced by cyanobacteria such as microcystin toxins and accumulate them in fish tissues (Mohamed et al., 2003; Deblois et al., 2008). Here in the present study, we did not investigate the cyanotoxins concentrations in fish tissues, but our previous studies reported the presence of microcystins in
Tilapia fish tissues at concentrations as high as $102 \mathrm{ng} \mathrm{g}^{-1}$ fresh weight (Mohamed et al., 2003). This may represent a risk to human health. Therefore, Nile tilapia fed on toxic cyanobacteria should be continuously monitored for microcystin levels in their tissues to ensure its suitability for human food. On the other hand, the inability of Tilapia fish to digest diatoms has been demonstrated earlier by Ping \& Jiankang (1994) and Grubach (2010) who found seventy-seven percent of the diatom taxa in the fish feces. This means that diatoms are more resistant to digestion in the fish gut than other phytoplankton groups. This resistance may be attributed to siliceous frustules which is hardly digested (Hamm et al., 2003), and may mechanically damage the intestinal cells (Giancamillo et al., 2012).

In conclusion, the results of this study along with other studies mentioned above, suggest a Nile Tilapia has high ingestion and digestion efficiencies for different forms of cyanobacteria (i.e. unicellular, colonies, filaments). Therefore, Tilapia may offer opportunities for control of harmful cyanobacteria in eutrophic lakes. However, cyanobacteria can produce cyanotoxins e.g., microcystins, and longterm exposure of Tilapia fish could increase the chances of toxin accumulation in fish tissues with potential transfer to higher trophic levels including human (Mohamed, 2016). Therefore, further studies are needed to determine levels of cyanotoxins in tilapia tissues from these fishponds and make an assessment of the potential health risk posed to humans living off this fishery.

\section{References}

ABDELGHANY, E. A. Food and feeding habits of Nile tilapia from the Nile River at Cairo, Egypt. Proceeding of the I International Conference on Fish Farm Technology, Trondheim, Norway, 1993, pp. 9-12.

ABDEL-TAWWAB, A.A. and SWEILUM, M.A. Improvement of fish production and water quality in tilapia cultured ponds by using different types of artificial diets and organic fertilizers. Veterinary Medical Journal Giza, 2003, 52, 19-28.

ABDEL-TAWWAB, M. and EL-MARAKBY, H. I. Length-weight relationship, natural food and feeding selectivity of Nile tilapia; Oreochromis niloticus (L.) in fertilized earthen ponds. In: R. BOLIVAR, G. MAIR, K. FITZSIMMONS eds. The 6th International Symposium of Tilapia in Aquaculture ISTA. Manila: Bureau of Fisheries \& Aquatic Resources, 2004, pp. 500-509.

ABDEL-TAWWAB, M. Food and feeding habits of Oreochromis niloticus under the effect of inorganic fertilizer with different N:P:K ratios in Abbassa 
fishponds. Egyptian Journal of Agricultural Research, 2000, 78, 437-448.

AWASTHI, M., DAS, N. and SINGH, R.K. Qualitative algal analysis from the fish-gut: Tested in the rice fish cropping system. Environmental Sciences, 2006, 3(1), 89-94.

BUDIHASTUTI, R., ANGGORO, S. and SAPUTRA, W. Analysis on the Feeding Habit of Tilapia (Oreochromis niloticus) Cultured in Silvofishery Pond in Semarang. Journal of Environmental Ecology, 2013, 4(2), 2157-6092. http://dx.doi.org/10.5296/ jee.v4i2.3950.

BWANIKA, G.N., CHAPMAN, L.J., KIZITO, Y. and BALIRWA, J. Cascading effects of introduced Nile Perch (Lates niloticus) on the foraging ecology of Nile tilapia (Oreochromis niloticus). Ecology Freshwater Fish, 2006, 15(4), 470-481. http://dx.doi.org/10.1111/ j.1600-0633.2006.00185.x.

DEBLOIS, C.P., ARANDA-RODRIGUEZ, R., GIANI, A. and BIRD, D.F. Microcystin accumulation in liver and muscle of tilapia in two large Brazilian hydroelectric reservoirs. Toxicon, 2008, 51(3), 435-448. http://dx.doi.org/10.1016/j. toxicon.2007.10.017. PMid:18067935.

FATTAH, I.M.S.A.E., AHMED, M.H. and AAL, M.A. Zooplankton as live food for fry and fingerlings of Nile Tilapia (Oreochromis niloticus) and Catfish (Clarias gariepinus) in concrete ponds. In: Proceedings of the VIII International Symposium on Tilapia in Aquaculture. Cairo: ISTA, 2008, pp. 757-771.

GIANCAMILLO, A.D., MARTINO, P.A., ARRIGHI, S. and DOMENEGHINI, C. Gut peculiarities of feed deprived white sturgeons (Acipenser transmontanus, Richardson 1836). Open Journal of Veterinary Medicine, 2012, 2(2), 52-59. http://dx.doi. org/10.4236/ojvm.2012.22009.

GRUBACH, P.G. The capacity of diatom species to survive ingestion by the algivorous minnow, peepholes notatus. Masters Theses, Essays, and Senior Honors Projects, 2010, 18, 137-141.

HAMM, C.E., MERKEL, R., SPRINGER, O., JURKOJC, P., MAIER, C., PRECHTEL, K. and SMETACEK, V. Architecture and material properties of diatom shells provide effective mechanical protection. Nature, 2003, 421(6925), 841-843. http://dx.doi.org/10.1038/nature01416. PMid:12594512.

KOMÁRKOVÁ, J. and TAVERA, R. Phytoplankton and equilibrium concept: the ecology of steady-state assemblages. Hydrobiolgia, 2003, 502(172), 187-196.

LU, K., JIN, C., DONG, S., GU, B. and BOWEN, S.H. Feeding and control of blue-green algal blooms by tilapia (Oreochromis niloticus). Hydrobiologia, 2006, 568(1), 111-120. http://dx.doi.org/10.1007/ s10750-006-0023-5.
MENEZES, R.F.,ATTAYDE, J.L. and VASCONCELOS, F.R. Effects of omnivorous filter-feeding fish and nutrient enrichment on the plankton community and water transparency of a tropical reservoir. Freshwater Biology, 2010, 55(4), 767-779. http://dx.doi. org/10.1111/j.1365-2427.2009.02319.x.

MOHAMED, Z. and AL SHEHRI, A.M. Microcystin production in epiphytic cyanobacteria on submerged macrophytes. Toxicon, 2010, 55(7), 1346-1352. http://dx.doi.org/10.1016/j.toxicon.2010.02.007. PMid:20167231.

MOHAMED, Z. and AL SHEHRI, A.M. Microcystinproducing bloom of Anabaenopsis arnoldi in a potable mountain lake In Saudi Arabia. FEMS Microbiology Ecology, 2009, 69(1), 98-105. http:// dx.doi.org/10.1111/j.1574-6941.2009.00683.x. PMid:19453492.

MOHAMED, Z.A. and AL-SHEHRI, A.M. Grazing on Microcystis aeruginosa and degradation of microcystins by the heterotrophic flagellate Diphylleia rotans. Ecotoxicology and Environmental Safety, 2013a, 96, 48-52. http://dx.doi.org/10.1016/j. ecoenv.2013.06.015. PMid:23856124.

MOHAMED, Z.A. and AL-SHEHRI, A.M. Assessment of cylindrospermopsin toxin in an arid Saudi lake containing dense cyanobacterial bloom. Environmental Monitoring and Assessment, 2013b, 185(3), 2157-2166. http://dx.doi.org/10.1007/ s10661-012-2696-8. PMid:22628106.

MOHAMED, Z.A. and BAKR, A. Concentrations of cylindrospermopsin toxin in water and tilapia fish of tropical fishponds in Egypt, and assessing their potential risk to human health. Environmental Science and Pollution Research International, 2018, 25(36), 36287-36297. http://dx.doi.org/10.1007/s11356018-3581-y. PMid:30368701.

MOHAMED, Z.A. Harmful cyanobacteria and their cyanotoxins in Egyptian fresh waters - state of knowledge and research needs. African Journal of Aquatic Science, 2016, 41(4), 361-368. http://dx.doi. org/10.2989/16085914.2016.1219313.

MOHAMED, Z.A., CARMICHAEL, W.W. and HUSSEIN, A.A. Estimation of microcystins in the freshwater fish Oreochromis niloticus in an Egyptian fish farm containing a Microcystis bloom. Environmental Toxicology, 2003, 18(2), 137-141. http://dx.doi.org/10.1002/tox.10111. PMid:12635102.

NATH, S.R., BERAKI, T., ABRAHA, A., ABRAHAM, K. and BERHANE, Y. Gut Content Analysis of Indian Mackerel (Rastrelliger kanagurta). Journal of Aquaculture and Marine Biology, 2015, 3(1), 1-5. http://dx.doi.org/10.15406/jamb.2015.03.00052.

OSTI, J.A.S., TUCCI, A. and CAMARGO, A.F.M. Changes in the structure of the phytoplankton community in a Nile tilapia fishpond. Acta 
Limnologica Brasiliensia, 2018, 30(0), e213. http:// dx.doi.org/10.1590/s2179-975x7917.

PING, X. and JIANKANG, L. Phytoplankton, especially diatoms, in the gut contents and feces of two plantivorous Cyprinids-silver carp and bighead carp. Chinese Journal of Oceanology and Limnology, 1994, 12(4), 308-315. http://dx.doi.org/10.1007/ BF02850490.

PRESCOTT, G.W. The algae: a review. Boston: Houghton Mifflin Co., 1978, 436 p.

RIVERA VASCONCELOS, F., MENEZES, R.F. and ATTAYDE, J.L. Effects of the Nile tilapia (Oreochromis niloticus L.) on the plankton community of a tropical reservoir during and after an algal bloom. Hydrobiologia, 2018, 817(1), 393-401. http://dx.doi. org/10.1007/s10750-018-3591-2.

SALAZAR TORRES, G., SILVA, L.H.S., RANGEL, L.M., ATTAYDE, J.L. and HUSZAR, V.L.M. Cyanobacteria are controlled by omnivorous filterfeeding fish (Nile tilapia) in a tropical eutrophic reservoir. Hydrobiologia, 2016, 765(1), 115-129. http://dx.doi.org/10.1007/s10750-015-2406-y.

SEMYALO, R., ROHRLACK, T., KAYIIRA, D., KIZITO, Y.S., BYARUJALI, S., NYAKAIRU, G. and LARSSON, P. On the diet of Nile tilapia in two eutrophic tropical lakes containing toxin producing cyanobacteria. Limnologica, 2011, 41(1), 30-36. http://dx.doi.org/10.1016/j.limno.2010.04.002.

SILVA, L.H.S., ARCIFA, M.S., SALAZAR-TORRES, G. and HUSZAR, V.L.M. Tilapia rendalli increases phytoplankton biomass of a shallow tropical lake. Acta Limnologica Brasiliensia, 2014, 26(4), 429-441. http://dx.doi.org/10.1590/S2179975X2014000400010.

TAVERA, K. Phytoplankton of tropical lake Catemaco [PhD Thesis]. České Budějovice, Czech Republic: University of South Bohemia; Faculty of biological science, $1996.62 \mathrm{p}$.

TEICHERT-CODDINGETON, D.R., POMA, T.J. and LOVSHINE, L.L. Attributes of tropical ponds cultured fish. In: H.S. ENGA and C.E. BOYD, eds. Dynamics of pond aquaculture. Boca Raton: CRC Press, 1997, pp. 183-198.

TURKER, H., EVERSOlE, A.G., ARNOLD, G. and BRUNE, D.E. Filtration of green algae and cyanobacteria by Nile tilapia, Oreochromis niloticus, in the Partitioned Aquaculture System. Aquaculture, 2003, 215(1-4), 93-101. http://dx.doi.org/10.1016/ S0044-8486(02)00133-3.

XIE, S., CUI, Y. and LI, Z. Dietary-morphological relationships of fishes in Liangzi Lake, China. Fish Biology, 2001, 58(6), 1714-1729. http://dx.doi. org/10.1111/j.1095-8649.2001.tb02325.x.

ZANDEN, V., SHUTER, M.J., LESTER, B.J. and RASMUSSEN, J.B. Within-and among-population variation in the trophic position of a pelagic predator, lake trout (Salvelinus namaycush). Canadian Journal of Fisheries and Aquatic Sciences, 2000, 57(4), 725-731. http://dx.doi.org/10.1139/f00-011.

Received: 15 December 2018 Accepted: 23 September 2019 